\title{
Involvement of interferon regulatory factor-1 in monocyte CD95 expression and CD95-mediated apoptosis
}

\author{
Cell Death and Differentiation (2003) 10, 615-617. doi: 10.1038/sj.cdd.4401213
}

\section{Dear Editor}

The control of CD95 expression is a key factor for the regulation of apoptosis in several cellular systems. Although different transcription factors, such as p53, SP-1, NF- $\kappa \mathrm{B}, \mathrm{GA}-$ binding protein and $A P-1$ have been implicated in the regulation of CD95 promoter in various cellular contexts, ${ }^{1-3}$ the molecular events regulating constitutive and inducible CD95 gene expression have not been fully elucidated. Interferon- $\gamma$ (IFN- $\gamma$ ) increases CD95 expression and CD95induced apoptosis in several cell types. ${ }^{4}$ We hypothesized that such upregulation of CD95 expression and signaling could be mediated by the interferon regulatory factor-1 (IRF1), a tumor-suppressor transcription factor involved in the immune-regulatory activity of interferons. IRF-1 is induced in response to IFN- $\gamma$ and to a variety of other cytokines, and has been shown to be involved in tumor suppression, growth regulation, cell differentiation and apoptosis. ${ }^{5-8}$ Several studies have contributed to define IRF-1 as a regulator of apoptosis: IRF-1 mRNA is induced by IFN- $\gamma$ earlier and more strongly than other apoptosis-related genes, suggesting a direct involvement of IRF-1 in the transactivation of proapoptotic gene families. Moreover, IRF-1 has been demonstrated to mediate DNA damage-induced apoptosis in mitogenactivated $\mathrm{T}$ lymphocytes, a process that does not require p53. ${ }^{9}$ Nevertheless, the specific role of IRF-1-inducible genes in IRF-1-dependent apoptotic pathway remains unknown. By using a dedicated computer program, we found two consensus binding sequences for IRF-1 in the human CD95 promoter sequence. In order to verify whether IRF-1 was able to bind the CD95 promoter, we synthesized two doublestranded oligonucleotides whose sequences reproduce the CD95 promoter region encompassing the two putative IRF-1binding sites. Both probes were ${ }^{32} \mathrm{P}$ labeled and utilized in electrophoretic mobility shift assay (EMSA) experiments with nuclear extracts obtained from U937 monocytic cells stimulated with IFN- $\gamma$ for $2 \mathrm{~h}$. In Figure 1a, we show the appearance of two inducible IRF-1-binding complexes, whose specificity was demonstrated by competition assays with a molar excess of unlabeled wild-type probe or of an unrelated oligomer. Moreover, an anti-IRF-1 polyclonal antibody was able to induce a supershift that could be abrogated by a specific neutralizing peptide. To assess whether IRF-1 binding plays a functional role in trans-activation of the human CD95 promoter, we performed the luciferase reporter gene assay in a recipient cell system using wild-type and mutated sequences derived from the CD95 promoter. As described in Figure $1 \mathrm{~b}$, the $C D 95$ promoter region from nt -1435 to $\mathrm{nt}$ +236 and a series of mutants were cloned upstream the luciferase gene in the PGL2 basic vector and the resulting constructs (PGL-CD95, mutant-1, -2 and -3 ) were transiently cotransfected in TK- ts 13 cells together with an IRF-1 expression vector ( $p A c t)$ or an empty control vector (pActC). The luciferase expression driven by the entire $C D 95$ promoter (PGL-CD95) was enhanced approximately 55 folds in the presence of the IRF-1 expression vector. The mutated CD95 promoter constructs $\mathrm{M} 1$ and $\mathrm{M} 2$, containing IRF-1-binding site 1 and IRF-1-binding site 2 , respectively, were both specifically trans-activated by IRF-1, although to a lesser extent (about 14 folds). As expected, the M3 construct, a deletion mutant without any IRF-1-binding site, was not substantially activated by IRF-1. In order to understand the role of IRF-1 in CD95 gene regulation, we analyzed the kinetics of IRF-1 and CD95 mRNA induction by IFN- $\gamma$ in peripheral blood-derived human monocytes and in U937 monocytic cells. After total RNA isolation, quantitative real-time RT-PCR was performed to evaluate IRF-1 and CD95 mRNA levels in cells stimulated up to $24 \mathrm{~h}$ with IFN- $\gamma$. Target genes (IRF-1 and CD95) were coamplified with the endogenous $18 \mathrm{~S}$ gene, and their levels were normalized on the basis of the endogenous control. As shown in Figure 1c, we observed a tight correlation between IRF-1 and CD95 gene expression levels both in monocytes and in U937 cells. This observation suggests the possibility that CD95 levels may be influenced by the amount of IRF-1 present in the cell. In order to investigate the effect of IRF-1 inhibition on CD95 expression, we performed a relative quantification of IRF-1 and CD95 transcript levels in monocytes or U937 cells treated with IRF-1 antisense oligodeoxynucleotides (ODN). In Figure 1d, we show that treatment of monocytes with antisense ODN for $24 \mathrm{~h}$ results in about $75 \%$ inhibition of IFN- $\gamma$-induced IRF-1 upregulation, with a consequent significant reduction of CD95 levels. Similar results were obtained in U937 cells. Moreover, the effect of IRF-1 inhibition on CD95 protein expression paralleled the effect on gene expression, as shown by cytofluorimetric analysis in Figure 1e. To investigate the possible role of IRF-1 in CD95mediated apoptosis, we targeted IRF-1 expression with antisense ODN and evaluated the levels of cell death induced by an agonistic anti-CD95 antibody in U937 cells. Cells were stimulated with IFN- $\gamma$ in the presence or absence of IRF-1 antisense or missense ODN and treated $4 \mathrm{~h}$ with anti-CD95 antibody. The percentage of apoptotic cells was determined by the Annexin V-propidium iodide standard assay. According to previous reports, we found that IFN- $\gamma$ stimulation increased CD95-mediated apoptosis of U937 cells. On the other hand, treatment with IRF-1 antisense ODN significantly inhibited CD95-induced cell death, whereas treatment with missense ODN did not reproduce the same effect (Figure 1f). 
These observations suggest that IRF-1 contributes to IFN- $\gamma$ mediated sensitization of monocytic cells to CD95-induced apoptosis.

CD95 induction by IRF-1, while not being the only event responsible for the proapoptotic activity of IRF-1, may contribute to explain the role of this transcription factor in determining susceptibility to cell death. Since IRF-1 has been demonstrated to mediate caspase-1 gene induction, the proapoptotic activity of IRF-1 has been in some cases ascribed to increased caspase- 1 expression and activation. ${ }^{10}$ However, whereas the importance of caspase-1 in the production of inflammatory cytokines is widely recognized, its role in apoptosis remains controversial and seems in most cases subordinated to caspase- 3 and -8 activation. It has been recently reported that the release of IFN- $\gamma$ by cytotoxic $T$ cells upregulates CD95 expression on the surface of target a
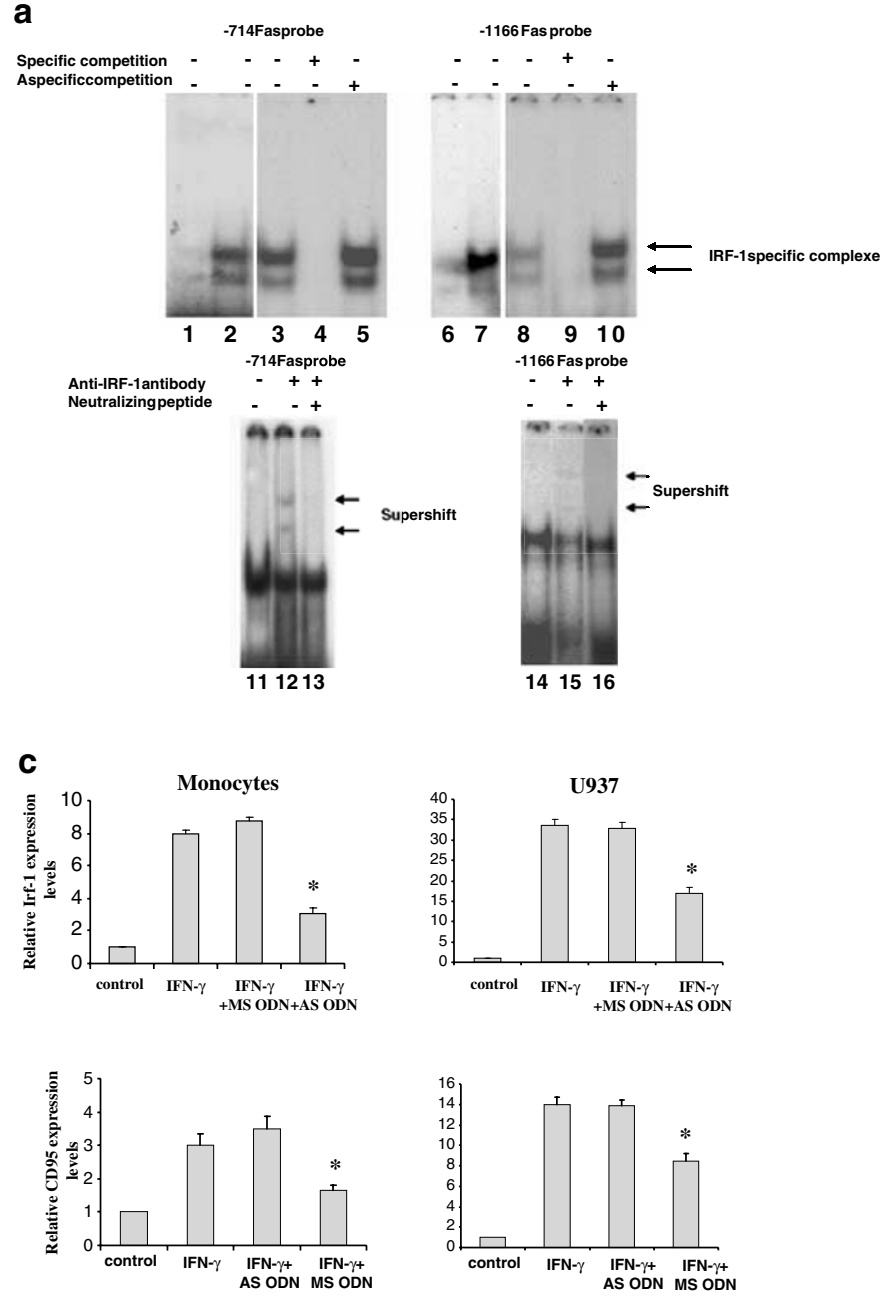

e
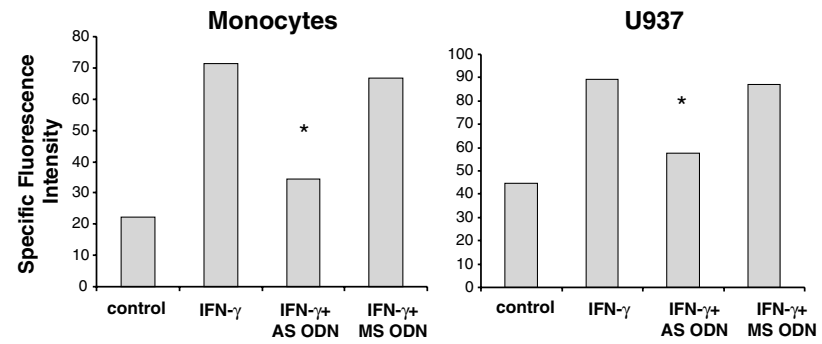

b

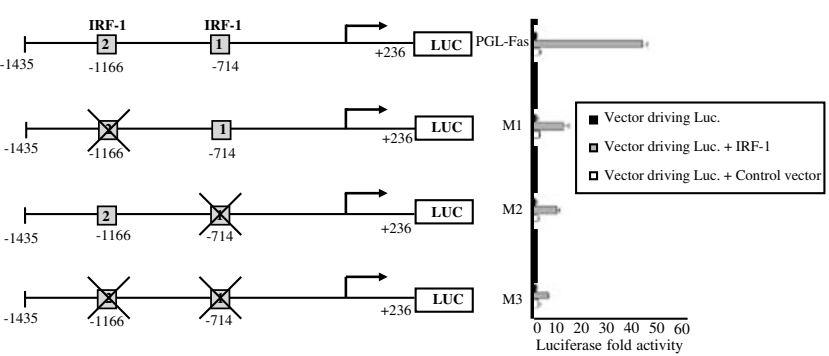

d

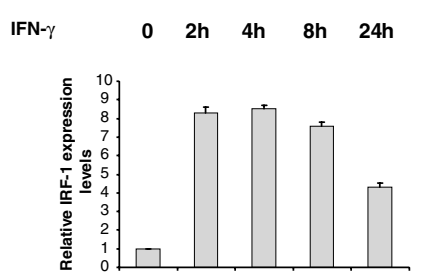

$\begin{array}{lllll}0 & 2 h & 4 h & 8 h & 24\end{array}$
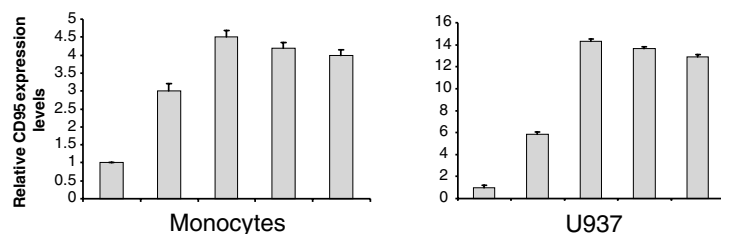

f

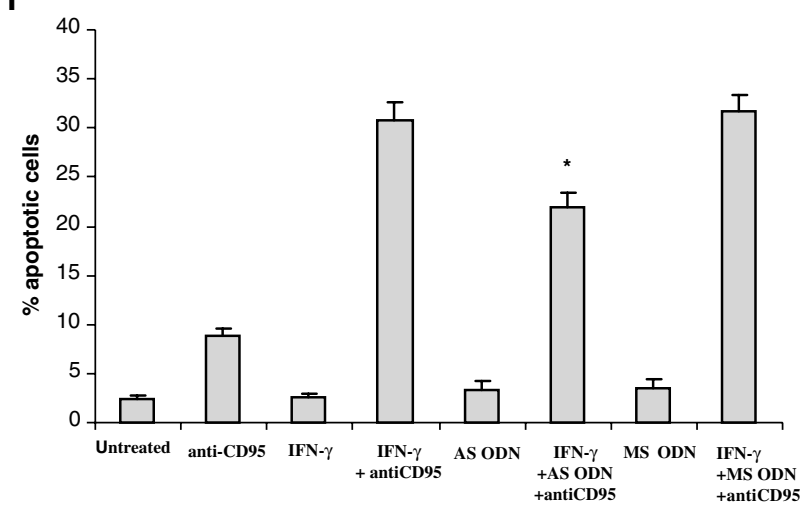


cells and facilitates perforin-independent target cell lysis. ${ }^{11}$ IFN- $\gamma$-mediated CD95 upregulation may play a role in the feedback regulation of an immune response by clearing APC that are no more necessary for antigen presentation. In this view, IFN- $\gamma$ released by CTL may activate IRF-1, which would contribute to CD95 upregulation and sensitization of monocytes to CD95-induced apoptosis. Since macrophages derived from IRF-1-/- mice are refractory to cytotoxicity induced by stimulation with LPS plus IFN- $\gamma,{ }^{12}$ it would be interesting to investigate the possible involvement of CD95 in this process. Our observation that IRF-1 is able to modulate CD95 levels may have potential implications also in nonhematopoietic cells, particularly where IRF-1 nullizygosity has been shown to determine an increased resistance to pathological cell death. IRF-1 expression is increased after cerebral ischemia in damaged regions of the brain, and IRF-1 knockout mice show a substantial reduction of infarctuated area and neurological deficits. Since CD95 has been reported to mediate ischemia/reperfusion-induced apoptosis, which involves death receptor-mediated damage, it would be interesting to evaluate the possible contribution of IRF-1 to the induction of CD95 expression in ischemic neurons. In conclusion, we provide evidence that IRF-1 plays a role in IFN- $\gamma$-induced CD95 expression and sensitivity of monocytic cells to CD95-mediated apoptosis. This study may contribute to clarify both the mechanisms that control CD95 expression and the links between IRF-1 and the transcriptional regulation of apoptosis.

\section{Acknowledgements}

We are grateful to Dr. Y Nakanishi and Dr. T Taniguchi for kindly providing CD95 promoter and IRF-1 expression vector, respectively. We thank Dr. Nicola Marziliano for helping in quantitative PCR measurements. A Zeuner and CG Messina are recipient of FIRC fellowships. This work was supported by AIRC grants.

\section{E Conte $^{\star 1}$, L Manzella ${ }^{1}$, A Zeuner ${ }^{1,2}$, G Cocchiaro ${ }^{1}$, C Conticello ${ }^{1}$, L Zammataro ${ }^{1}$, CG Messina ${ }^{2}$, R De Maria ${ }^{2}$ and $A$ Messina ${ }^{1}$}

\footnotetext{
1 Department of Biomedical Sciences, University of Catania, Italy

2 Department of Hematology and Oncology, ISS, Rome, Italy

* Corresponding author: E Conte, Department of Biomedical Sciences, via Androne 83, Catania 95124, Italy. Tel: +39 095 313429; Fax: +39 095 320267; E-mail: econte@unict.it
}

1. Chan $\mathrm{H}$ et al. (1999) Mol. Cell. Biol. 19: 2098-2108

2. Owen-Schaub et al. (1995) Mol. Cell. Biol. 15: $3032-3040$

3. Li XR et al. (1999) J. Biol. Chem. 274: 35203-35210

4. Ossina NK et al. (1997) J. Biol. Chem. 272: 16351-16357

5. Willman CL et al. (1993) Science 259: 968-971.

6. Harada $\mathrm{H}$ et al. (1993) Science 259: 971-974.

7. Tanaka N, Kawakami T, Taniguchi T (1993). Mol. Cell. Biol. 13: 4531-4538.

8. Liebermann DA, Hoffman B (1994) Stem Cells. 12: 352-369.

9. Tamura $T$ et al. (1995) Nature 376: 596-599.

10. Kim EJ et al. (2002) J. Cell. Biochem. 85: 369-380

11. Mullbacher A et al. (2002) J. Immunol. 169: $145-150$

12. Iadecola C et al. (1999) J. Exp. Med. 189: 719-727

Figure 1 (a) In vitro analysis of two potential IRF-1-binding sites on the CD95 promoter. EMSA with nuclear extracts from U937 cells unstimulated (lanes 1 and 6 ) or stimulated for $2 \mathrm{~h}$ with $200 \mathrm{U} / \mathrm{ml} \mathrm{IFN}-\gamma$ (lanes 2-5 and 7-10), incubated with the two ${ }^{32}$-labeled CD95 probes corresponding to IRF-1-binding site 1 (-714) and 2 $(-1166)$. The specific IRF-1 complexes are indicated by arrows. Specificity was demonstrated by competition with 100 -fold molar excess of cold CD95 probes (lanes 4 and 9) or with 100-fold molar excess of aspecific (Oct2A) probes (lanes 5 and 10). For supershift analysis with anti-IRF-1 antibody, nuclear extracts of U937 cells stimulated for $2 \mathrm{~h}$ with $200 \mathrm{U} / \mathrm{ml}$ IFN- $\gamma$ incubated with the two ${ }^{32} \mathrm{P}$-labeled CD95 probes (lanes 11 and 14); the same nuclear extracts incubated in the presence of $1 \mu \mathrm{g}$ of anti-IRF-1 antibody alone (lanes 12 and 15) or in combination with $1 \mu \mathrm{g}$ of neutralizing peptide (lanes 13-16). These results are representative of three independent experiments with similar results. (b) CD95 promoter-luciferase constructs and functional study of IRF-1 activity on CD95 promoter by luciferase gene assay in recipient cells. Schematic representation of CD95 promoter-luciferase constructs used for transient transfection and luciferase assay. Location of the two putative IRF-1-binding sites is indicated. Luciferase fold activity of CD95-luciferase constructs alone (black bars), in the presence of IRF-1 expression vector pAct (gray bars) or empty vector pActC (white bars), are indicated in the diagrams. Average data with S.D. are the result of triplicate experiments. (c) Quantitative real-time RT-PCR analysis of IRF-1 and CD95 gene expression in monocytic cells stimulated for various times with IFN- $\gamma$. Peripheral blood-derived human monocytes and U937 cells were stimulated with $200 \mathrm{U} /$ $\mathrm{ml}$ IFN- $\gamma$ or the times indicated. Quantitative real-time RT-PCR was performed with Abi Prism 7700 (PE Applied Biosystems) following the manufacturer's instructions. Primers for IRF-1 were: IRF1F: 5'-CATGGCTGGGACATCAACAA-3', IRF1R: 5'-GTTCATGGCACAGCGAAAGTT-3': The sequence of TaqMan 5' FAM-labeled IRF-1 probe was: $5^{\prime}$-TGTTCCGGAGCTGGGCCATTCAC-3'. Primers and TaqMan probes for CD95 and endogenous $18 \mathrm{~S}$ were Pre-Developed TaqMan Assay Reagents (PE Applied Biosystems). All reactions were performed by coamplifying in the same tube endogenous and target genes, and relative quantitative evaluations of target levels were performed with the dedicated PE Applied Biosystems software. Diagram bars represent IRF-1 and CD95 expression levels normalized on the basis of 18 S values, assuming as one unit IRF-1 and CD95 levels of untreated cells. Average and S.D. of triplicate measurements of two independent experiments are reported. (d) Effect of IRF-1 antisense oligonucleotides on IRF-1 and CD95 gene expression in monocytic cells stimulated with IFN- $\gamma$. Peripheral blood-derived human monocytes and U937 cells were stimulated with $200 \mathrm{U} / \mathrm{ml} \mathrm{IFN}-\gamma$ for $4 \mathrm{~h}$ in the presence of antisense or missense ODN ( $1 \mu \mathrm{mol} / \mathrm{l})$. Quantitative real-time PCR was performed as previously described. Averages and S.D. of triplicate measurements of two independent experiments are reported. Statistical analysis was performed by Student's $t$-test, ${ }^{*} P<0.05$ versus IFN- $\gamma$. (e) Effect of IRF-1 antisense oligonucleotides on CD95 protein expression in monocytic cells stimulated with IFN- $\gamma$. Peripheral blood-derived human monocytes and U937 were stimulated with $200 \mathrm{U} / \mathrm{ml} \mathrm{IFN}-\gamma$ for $24 \mathrm{~h}$ in the presence of antisense ODN or missense ODN (1 $\mu$ mol/I) and incubated with purified mouse antihuman CD95 IgG1 monoclonal antibody or mouse IgG1 control. Cells were subsequently washed and treated with PE-conjugated goat anti-mouse IgG antibody. Specific fluorescent intensities of individual cells were determined by dividing mean fluorescence intensity of anti-CD95-stained cells with mean background fluorescence intensity obtained with isotypic lgG1. Average values of two independent experiments are reported. Statistical analysis was performed by Student's $t$-test, ${ }^{*} P<0.05$ versus IFN- $\gamma$. (f) Effect of IRF-1 antisense oligonucleotides on CD95-mediated apoptosis in monocytic cells. U937 cells were incubated $12 \mathrm{~h}$ with $200 \mathrm{U} / \mathrm{ml}$ IFN- $\gamma$ in absence or presence of IRF-1 antisense ODN or missense ODN ( $1 \mu \mathrm{mol} / \mathrm{l})$. Untreated and treated cells were stimulated with an agonistic anti-CD95 antibody (100 $\mathrm{ng} / \mathrm{ml})$ for $4 \mathrm{~h}$. Apoptotic cells were evaluated as Annexin-V positive cells by flow-cytometric analysis following Annexin-V, propidium iodide staining by Sigma kit. Averages values and S.D. of three independent experiments are reported. Statistical analysis was performed by Student's $t$-test, ${ }^{\star} P<0.05$ versus IFN- $\gamma+$ antiCD95 